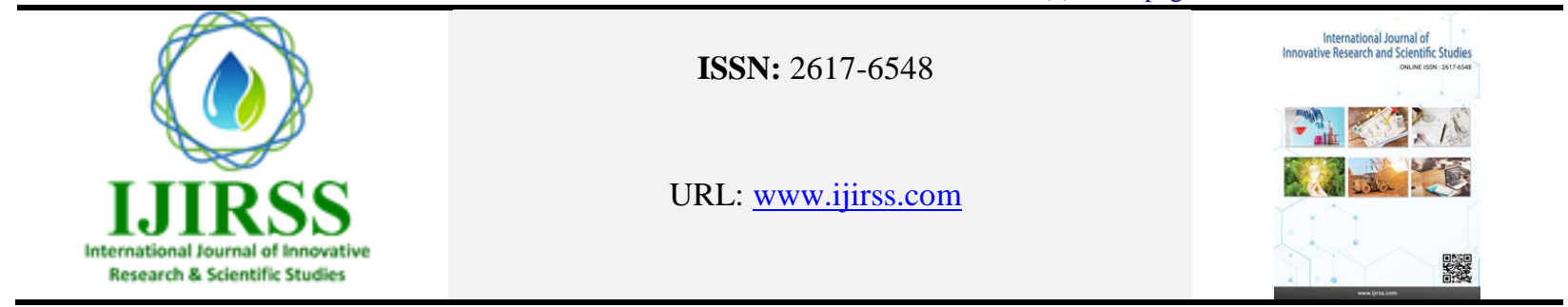

\title{
A Review on Pathophysiology and Pharmacological Treatment of COVID-19
}

\author{
Nelofar Sediqi ${ }^{*}$, Maryam Sediqi $^{2}$ \\ ${ }^{1}$ Department of Pharmacology, Faculty of Pharmacy, Kabul University, Kabul, Afghanistan \\ ${ }^{2}$ National Medicine Health Regulatory Authority, Quality Control of Medicine and Health Care Products, Ministry of Public Health, \\ Kabul, Afghanistan \\ *Corresponding author: Nelofar Sediqi (sediqinilofar9@gmail.com)
}

\begin{abstract}
A cluster of patients with unidentified pneumonia cause emerged among people in a city of China named Wuhan, at the end of 2019, which then by laboratory analysis, was considered to be caused by a novel coronavirus (CoV) called 2019-nCoV. It has been declared a pandemic by the World Health Organization on March 11, 2020. By the spread of pandemic novel coronavirus, it is revealed that severe acute respiratory syndrome coronavirus-2 (SARS-CoV-2) is genetically related to SARS-CoV of the 2003 outbreak. However, the latest study showed that angiotensin-converting enzyme- 2 is the receptor used by SARS-CoV-2 to enter into the human cells, alike its predecessor (SARS-CoV). Both excessive immune reactions and lytic effects of the virus on host cells play a central role in the pathogenesis of COVID-19. Commonly, the antivirals, corticosteroids, antibiotics, Immunoglobulin, and some other drugs are used as a pharmacological treatment in COVID-19 patients. Recently, the food and drug administration (FDA) has authorized the emergency use of remdesivir for the treatment of COVID-19 under an emergency use authorization (EUA). The majority of the current drugs are recommended based on the treatment of previous epidemic viruses. Due to its pandemic prevalence, and the insufficient of specifically approved drugs and vaccines, COVID-19 is becoming an emergency public health concern. Furthermore, there is a lack of comprehensive knowledge about pathogenesis and treatment of COVID-19. Thus, this review answers to the questions about pathogenesis and pharmacological treatment of COVID-19 and provide the newest information in term of the pathogenesis and pharmacological treatment of COVID-19, inclusively, and simultaneously.
\end{abstract}

Keywords: SARS-CoV-2, COVID-19, Pathophysiology, Pharmacological treatment.

DOI: $10.53894 /$ ijirss.v3i2.37

Funding: This study received no specific financial support.

History: Received: 6 May 2020/Revised: 26 May 2020/Accepted: 11 June 2020/Published: 18 June 2020

Licensed: This work is licensed under a Creative Commons Attribution 4.0 License (cc) EY

Acknowledgement: Both authors contributed to the conception and design of the study.

Competing Interests: The authors declare that they have no conflict of interests.

Transparency: The authors confirm that the manuscript is an honest, accurate, and transparent account of the study was reported; that no vital features of the study have been omitted; and that any discrepancies from the study as planned have been explained.

Ethical: This study follows all ethical practices during writing.

\section{Introduction}

Coronavirus generally depends on the Coronaviridea family, and the Nidovirales order [1]. They are enveloped with positive sense-single-stranded ribonucleic acid (RNA), with a characteristic appearance that resembles a crown (corona in Latin means crown) due to the presence of club-shaped surface protein projections [2]. The first cases of coronavirus 
outbreak spread from a city in China named Guangdong in late 2002 with more than 300 cases and five deaths. This epidemic which named severe acute respiratory syndrome (SARS) then had spread to many countries, mostly in Asia, but also to Toronto, Canada [1]. At the end of 2019, pneumonia with unidentified cause begins among people in a city of China named Wuhan, which then by laboratory analysis, unidentified pneumonia was considered to be caused by a novel coronavirus (CoV) called 2019-nCoV [3]. By the spread of pandemic novel coronavirus, it is revealed that SARS-CoV-2 is genetically related to SARS-CoV of the 2003 outbreak [1]. However, the latest study showed that angiotensin-converting enzyme-2 (ACE2), a membrane exopeptidase, is the receptor used by SARS-CoV-2 to enter into the human cells, alike its predecessor (SARS-CoV). The incubation period for CoVs may last from two to seven days, clinical sings contain high fever, chills, cough, shortness of breath or difficulty in breathing, diarrhea, myalgia or fatigue, expectoration, and hemoptysis. In severe cases may develop pneumonia, and severe conditions such as heart failure, respiratory failure, and liver failure can occur in elderly patients [2]. Evidence from the first cases showed that the animal to human transmission assumed as a primary mechanism of transmission. However, due to the globally spreading of COVID-19, human to human transmission is the major mechanism of transmission [4]. Globally, as of June 7, 2020, there have been 6,750,521 confirmed cases of COVID-19, including 395,779 deaths as WHO report [5]. A diverse strategy of treatment such as nonpharmacological therapy, pharmacological therapy, and psychological therapy are operated for managing the COVID-19 patients, and various studies are in progress for discovering specific new agents for the treatment of COVID-19 in several countries [6]. Most of the current drugs are recommended based on the treatment of previous epidemic viruses such as malaria, ebola, severe acute respiratory syndrome (SARS), and the middle east respiratory syndrome (MERS) [7]. Due to its more prevalence and the lack of specifically approved drugs, COVID-19 is becoming an emergency public health concern. Therefore, this review will provide a piece of comprehensive knowledge about pathogenesis and pharmacological treatment of COVID-19.

\section{Pathophysiology}

SARS-CoV-2 is a positive single-strand RNA virus that structurally contains an envelope, nucleocapsid, spike glycoproteins on the envelope, and other non-structural proteins. Both structural proteins (spike, membrane, envelope, and nucleocapsid proteins) and non-structural proteins play key roles in pathophysiology and virulence mechanism in SARSCoV-2. Studies have shown that non-structural proteins inhibit the inherent immune reactions and among structural proteins, envelope plays a central role in virulence of SARS-CoV-2, and it improves the accumulation and release of the virus. Spike glycoproteins are another essential structural proteins that direct the binding of the virus to host receptors ACE-2, [4]. The first stage for coronavirus to infect host cells is the attachment of its S-spike protein and ACE2 receptors, but before the binding of the spike protein, the spike protein is activated by the transmembrane serine protease (TMPRSS)[8]. The spike protein of coronavirus contains two domains, the S1, which is important in receptor attachment and the S2, which plays a role in plasma membrane fusion [9]. ACE2 provides a direct binding site for the S protein of CoVs. The sites of the receptor-binding domain (RBD) is located at the C-terminus of S1 of S-spike protein. Besides spike proteins, envelop protein, membrane glycoprotein, nucleocapsid protein and genomic RNA of the virus play key roles in the pathogenesis of the COVID-19 [3]. ACE2 is a type 1 membrane protein member of the ACE family which is expressed by the epithelial cells of the lungs, kidney, gastrointestinal tract, and cardiovascular system. Primarily binding of S-spike protein with ACE2 receptors, within lung cells, are causing the severe acute respiratory syndrome and damaging of other organs [10]. In the pathogenesis of COVID-19, both excessive immune reactions and lytic effects of the virus on host cells will play the central role. Studies have shown in the initial stage of the illness, the patients suffering from common symptoms such as severe pneumonia, fever, and dry cough. In some patients, the illness forward to acute respiratory stress syndrome (ARDS) and septic shock, which was finally, followed by multi-organ dysfunction, and about $10 \%$ of patients have died [9]. Older age, previous diseases such as hypertension, diabetes, and coronary artery disease are the most important risk factors for death $[10,11]$. The results of a retrospective study have shown that according to the severity the COVID-19 has displayed different stages of the disease from mild in 38\% of the patients, to sever in $35 \%$ of patients and critical in $28 \%$ of the patients [12].

\subsection{Hyperactivation of Immune System}

Immune cells play a crucial function in terms of pathogens such as bacteria, viruses, and fungi, and so on. Clinical studies showed that immune system response during SARS-CoV-2 infection leads to autoimmune disease.

Mechanism: Viral rapid replication induced immune cells response which leads to hyperactivation of T-cells that besides infected cells, healthy cell damage occurs by high concentrations of peripheral CD4 and CD8 T cells. Once type 2 pneumocyte broke, it may attract macrophages. After activation of macrophages, specific cytokines including interleukin 1 (IL - 1), interleukin 6 (IL - 6), and tumour necrosis factor-alpha (TNF - a) are released. They mediate proteins moving into the bloodstream, which caused blood vessel dilation on endothelial cells of the microvasculature around the alveoli which then leads to an increase in the permeability of blood vessels around the alveoli. The fluids accumulation as a result of blood vessel permeability leads to compressive pressure which makes it difficult to breathe and consequently, respiratory distress. On the other hand, the production of surfactant is decreased because of type - 2 pneumocyte death that increases alveolar surface tension. An increase in alveolar surface tension causes alveolar collapse, which ends up in respiratory distress. Besides these immune cells response, neutrophil activation occurs as well to neutralize the virus by releasing reactive oxygen species (ROS) and proteases. As rapid RNA replication happens, excessive ROS and protease are produced, which not only neutralize the viruses but also attack the cells lining alveoli. In this case, type 1 and type 2 
pneumocytes lose their function, which infected persons suffer severe hypoxia and pneumonia [3]. Hyperactivation of the immunity system also caused systemic cellular damaging that leads to multi-organ dysfunction $[3,10]$.

\subsection{Sever Pneumonia}

WHO reported that severe pneumonia is the most common disorder of severing COVID-19 [13]. Severe pneumonia is linked with serious dyspnoea, respiratory distress, and hypoxia. Nevertheless, the fever has to be cautiously clarified because in a sever stage, also it should be moderate or absent [4].

Mechanism: As studies have shown the pathogenesis of pneumonia might be complicated and might be linked with hyperactivation of the host immune system. SARS-CoV-2 can activate the extreme immune response that sometimes the "cytokine storm" and multi-organ dysfunction happened respectively [14]. The inflammation and host immune system response caused the fluids and pus accumulation into alveoli which person is not able to inspire adequate oxygen. Thus, may other organ disorders such as kidney, heart, and others happened. Older people, people with a history of respiratory system disorders and previous disease might be more susceptible [13].

\subsection{Acute Respiratory Distress Syndrome (ARDS)}

It is a life-threatening stage; it may be a sudden severe respiratory failure or impairing of old respiratory symptoms. It has different stages, such as mild, moderate, and severe [4].

Mechanism: The main target cell for the SARS CoV-2 is pneumocytes type - 2 (alveoli are lined with two types of pneumocytes which type 1 is responsible for gasses exchange while type - 2 is responsible for the production and secretion of surfactant, the molecule that reduces the surface tension of pulmonary fluids and makes it easy to breath). The problem begins with the binding of S- spike protein of the virus and ACE-2 on the surface of pneumocyte type - 2 (this process is triggered by the S1 subunit shedding and S2 subunit transition of S protein). After binding, it enters the cytoplasm of the pneumocyte where the RNA of the virus is released. This positive sense single-stranded RNA acts as mRNA and translated proteins. One of the translated proteins of SARS-CoV-2 is the RNA-dependent-RNA polymerase that leads to the synthesis of multiple RNA copies. Due to this mechanism, hundreds and thousands of viral particles arise within the cell. As normally, ACE2 breaks down angiotensin-2 into vasodilator angiotensin 1-7 which ACE2/Ang 1-7 plays an antiinflammatory and antioxidant role in the lungs against ARDS. Excessive binding of SARS-CoV-2 with the ACE2 receptor leads to the significant reduction of enzymatic activity of ACE2, downregulation of ACE2 receptors makes them unable to protect against lung injury as the enzyme gets degraded by the virus [10].

\subsection{Septic Shock}

Sepsis and septic shock are associated with bloodstream infections. Sepsis is a result of the potential response of the host immune system against infections. SARS-COV-2 in sever stage caused life-threatening septic shock which includes a variety of signs and symptoms such as serious dyspnea and hypoxia, kidney disorder with oliguria, tachycardia, mental disorder, acidosis and others [8]. The results of the retrospective cohort study described the Sepsis one of the common complications in COVID-19 patient, which SARS-CoV-2 infection might be directly linked to it. However, more study is required to clarify the pathogenic mechanism of SARS-CoV-2's sepsis. Additionally, the results indicated that more than $50 \%$ of patients developed sepsis [9].

\subsection{Fever and Cough}

The release of a high amount of immune cells makes it easy for IL - 1 and IL - 6 seep into the bloodstream and travel to the central nervous system. In this case, prostaglandins are released by the hypothalamus of the central nervous system in responding to the increased concentration of these mediators. As a result, body temperature is increased, which fever is a significant symptom of COVID-19. Cellular debris in the alveoli needs to be moved out of the lungs, which, due to moving out of this debris coughing happens, which is a common symptom of COVID-19 [10].

The results of a study in china that was supported by the National Health Commission of China in 1099, COVID-19 patients described both the fever and cough as the most common symptoms. The cough was present in $67.8 \%$ of patients, and the fever was $43.8 \%$ on admission time, and it progressed up to $88.7 \%$ during hospitalization [15].

\section{Treatment}

As mentioned, the COVID-19, according to the severity, has several steps; hence the management performs according to the clinical manifestation and the severity. So, patients who have mild clinical manifestation (dyspnea not present), home management is adequate. Certain attention should pay for asymptomatic patients who are the main source of infection and can transmit the infection to others. Patients who are in moderate and severe stages of infection require hospitalization and supportive care (oxygen-therapy, a mechanical ventilator for ARDS) [16]. Isolation is the core management of COVID-19 for the prevention of healthy people and keeping the new positive cases at a low rate. Currently, there is not available any specific drugs or vaccine for treatment and prevention of COVID-19, and the major intervention involves symptomatic therapy and oxygen therapy [4]. However, there are some potential therapeutics that are under investigation for treating the COVID-19. Among these drugs, some of them are approved and use for other diseases, but they are an indication for COVID-19 require several studies. 


\subsection{Non-Pharmacological Therapy}

Non-pharmacological therapy is the main strategy for management of the COVID-19 which involves the general cares such as proper nutrition, adequate electrolytes, fluids, controlling the vital signs and laboratory biomarkers (neutrophil count, inflammatory cytokine and lymphocyte count, C-reactive protein and so on), symptomatic therapy and the supportive cares [17].

Symptomatic treatment: COVID-19, like other infections, has the symptom of the fever as the main symptom. Administration of the antipyretic and cooling methods recommended by WHO for managing the COVID-19 fever and the fever complications. COVID-19 patients who have other diseases such as hypertension and diabetes are more at the risk of the COVID-19 severity. Thus, continuous controlling of the blood sugar and blood pressure and proper actions are required. COVID-19 mediated multi-organ dysfunction is another complication that requires certain operations [17].

Supportive treatment: Several investigations determined an excessive need for respiratory support and oxygen therapy in severe cases of COVID-19 [17]. WHO recommends the high-flow nasal oxygen (HFNO) or non- invasive ventilation (NIV) and protective mechanical ventilation for managing of the respiratory failure [4]. Tracheostomy also may be performed in patients who require prolonged mechanical ventilation and longer intensive care unit (ICU) stay [2].

Psychological support: as COVID-19 brings a general change in humans life; thus, the psychological support for the patients, the patient relatives, and health workers may have a key impact on managing this pandemic. These psychological supports should provide helpful online recommendations by expert psychologists to whole people. Using hypnotic drugs in COVID-19 patients may affect the quality of sleep and the utilization of oxygen. Psychological supports also plays a central role in removing the fear and anxiety related to the COVID-19 among the whole of the people [17].

\subsection{Pharmacological Therapy}

Various group of medications is used for the treatment of COVID-19 patients which among them antiviral agents, antibiotics, corticosteroids, and intravenous immunoglobulin are the major classes. But to identify which group of medications are may effective or may not be still diverse studies are in progress [17].

\section{Antivirals}

Starting an early antiviral therapy can reduce the progression of mild cases to severe and critical cases [18].

Ribavirin: is a ribonucleoside analogue that is active against some coronaviruses and used as a supportive medicine in the treatment of SARS-COVID-19. Ribavirin inhibits viral RNA-dependent RNA polymerase [19]. During the outbreak of SARS in Hong Kong, ribavirin was used for patients [3] as its activity against SARS-CoV was limited and required high concentrations to inhibit viral replication in vitro, necessitating high dose (e.g., $1.2 \mathrm{~g}$ to $2.4 \mathrm{~g}$ orally every 8 hours) and combination therapy. For this reason, its usage has become limited in the treatment of COVID-19, if used, combination therapy may have clinical efficacy [19]. Therefore, the combined usage of Ribavirin with Interferon (IFN) beta could synergistically inhibit SARS-associated CoV replication by inhibiting RNA-dependent RNA polymerase in vitro [3]. However, the efficacy in COVID-19 patients still needs to be confirmed by clinical trials. Clinically, an appropriate dosage of ribavirin should be given to avoid an adverse reaction, including dose-dependent anemia [20].

Lopinavir/Ritonavir: The combination of lopinavir/ritonavir is used in the treatment of human immunodeficiency virus (HIV) infection. As the usage of these two medicines with ribavirin has a good therapeutic effect in SARS and MERS [3]. Therefore, lopinavir/ritonavir can be recommended for the treatment of novel coronaviruses in clinical trials via inhibition of 3-chymotrypsin-like protease in vitro [19], to inhibit viral RNA synthesis [20]. Recent studies indicates a delay in the start of treatment, which this effect may partially explain the ineffectiveness of lopinavir/ritonavir for the treatment of COVID-19. Some adverse effects should be considered, including gastrointestinal distress such as nausea and diarrhoea (up to 28\%) and hepatotoxicity (2-10\%) [19]. It also can increase the risk of cardiac arrhythmias (e.g..QT Prolongation) and significant drug interactions [21]. The recommended dosing regimen for lopinavir is $400 \mathrm{mg}$ twice each day, and ritonavir $100 \mathrm{mg}$ twice each day for up to 14 days [2].

Nelfinavir: It is another antiviral drug that works as a selective inhibitor of HIV protease, which has been effective in inhibition of SARS-Cov and a possible therapeutic option for SARS-COV-2 [3].

Umifenovir (Arbidol): Arbidol and its derivative, arbidomesylate, have been reported to have antiviral activity against SARS-CoV as well as against influenza viruses by blocking viral fusion [3]. As it targets S protein/ACE interaction by inhibiting membrane fusion of the viral envelop [22]. According to these data, clinical trials have been started in the treatment of SARS-CoV-2 patients [23]. The current dose of $200 \mathrm{mg}$ orally every 8 hours for influenza is being studied for COVID-19 treatment [19].

Remdesivir: It is an antiviral drug that inhibits RNA-direct RNA polymerase of coronaviruses in vivo and in vitro, inhibiting the replication of both endemic and zoonotic strains in cell culture [20]. Studies show that remdesivir inhibits the replication of both SARS-CoV and MERS-CoV as well as improve viral lung load, a clinical sign of lung disease, and respiratory function in the mouse model in vitro [23]. Based on its anti-CoVs activity, remdesivir was supplied by the manufacturer for experimental use in china, to treat hospitalized patients of COVID-19. In late February, its safety and efficacy are evaluated in hospitalized adults diagnosed with COVID-19 has started at the University of Nebraska medical centre in Omaha in the US [20]. It is approved that the antiviral protection of remdesivir and IFN beta are superior to that of lopinavir/ritonavir-IFN beta against MERS-CoV in vitro and in vivo [3]. It was also used in the treatment of ebola virus infections [10]. Furthermore, remdesivir was used for the treatment of the first COVID-19 patient in the United State, and its antiviral activity has been recommended against SARS-CoV-2 in vitro. Remedisivir, a broad spectrum antivirus has demonstrated in vitro and in vivo efficacy against SARS-CoV-2 as also initiated its clinical trial [9]. Recent studies have 
shown its efficacy in which several clinical trials are recruiting patients to evaluate the clinical efficacy of remdesivir for treatment of COVID-19 by using the dosage regimen of $200 \mathrm{mg}$ loading dose on the first day, following by $100 \mathrm{mg}$ intravenous once-daily maintenance dose for 9 days [23]. The Washington Department of Health administrated remedisivir intravenously first and found that remedisivir might have potential protection from SARS-CoV-2 infection [6]. Thereby, the FDA has authorized the emergency use of remdesivir for the treatment of COVID-19 under an EUA [24, 25].

Favipiravir: It is a nucleoside analog that is used in treating influenza A and B as well as identified as a potential broad-spectrum antiviral for COVID-19. It works by inhibiting RNA-directed RNA-polymerase of various RNA viruses [20]. Favipiravir inhibits in vitro replication of a wide range of influenza viruses and many other RNA viruses including arenaviruses, bunyaviruses, flaviviruses, alphaviruses, paramyxoviruses, and norovirus family. It also suppressed the replication of the zaire ebolavirus (EBOV) in vitro. Based on these data, patients hospitalized with COVID-19 are being recruited for a clinical trial evaluating the efficacy of favipiravir. The regimen does used in COVID-19 trials were mostly based on the dosing approved for influenza treatment, for example, $1600 \mathrm{mg}$ twice daily on the first day, following by 600 mg twice daily for six days [23]. Also, its teratogenicity and early embryonic death effects have been observed in animal studies, which should be considered [21].

Oseltamivir: It is used to inhibit the viral neuraminidase, which blocks the release of viral particles from the host cell by reducing the spread of viral in the respiratory tract. It is mostly used for the treatment of influenza A and B. Furthermore, it is reported that oseltamivir is used in the treatment of the COVID-19 epidemic in China, either with or without the use of antibiotics and corticosteroids. In addition, oseltamivir is used in a clinical trial with multiple combinations with chloroquine and favipiravir which has shown antiviral activity against SARS-CoV-2 and low toxicity [22]. However, Neuraminidase inhibitors do not appear to have activity against COVID-19, and the role for oseltamivir specifically for COVID-19 is limited, and additional clinical trials are required to demonstrate its antiviral activity against SARS-CoV-2 [26].

\subsection{Chloroquine/Hydroxychloroquine}

Chloroquine and hydroxychloroquine are FDA-Approved antimalarial drugs. They are used as antiviral drugs in the treatment of HIV, which they are acted by inhibiting virus entry into host cells. Chloroquine is used to inhibit the internalization of the virus through binding the ACE2 receptor and taken up by the cells as a vacuole [19]. It can inhibit COVID-19 in vitro and is recommended for the clinical control of viral replication [3]. Studies have shown that chloroquine and hydroxychloroquine block viral entry into cells by inhibiting glycosylation of host receptors, proteolytic processing, and endosomal acidification. These agents also have immunomodulatory effects through attenuation of cytokine production and inhibition of autophagy and lysosomal activity in host cells. A recent study in French on 36 patients (20 in the hydroxychloroquine group and 16 in the control group) reported virologic clearance improvement with the use of hydroxychloroquine, $200 \mathrm{mg}$ every 8 hours compared with control patients receiving standard supportive care. Virologic clearance at day 6, measured by nasopharyngeal swabs, was $70 \%$ (14/20) vs. (2/16) for the hydroxychloroquine and control groups, respectively. It is also suggested that a combination of azithromycin with hydroxychloroquine revealed superior viral clearance $6 / 6,100 \%$ ) compared with hydroxychloroquine monotherapy. This study was not able to support completely its safety and requires additional studies. Furthermore, another study estimated as well that $100 \%$ of patients treated with hydroxychloroquine in combination with the macrolides antibiotic azithromycin were virologically cured compare to $57.1 \%$ in patients treated with hydroxychloroquine alone [22]. Both agents can cause some serious adverse effects $(<10 \%)$, including QTc prolongation, hypoglycemia, neuropsychiatric effects, and retinopathy [19]. Besides, because of their antimicrobial nature make many researchers able to suggest chloroquine and hydroxychloroquine in the treatment of SARS-COVID-19. Chloroquine presumably alters the $\mathrm{pH}$ at the surface of the alveolar cells, thereby inhibiting the binding of SARS-CoV-2 to ACE2, which prevents the entry of the virus into the cells [10]. Also, it is considered that hydroxychloroquine has a favourable safety profile to chloroquine though they are equally effective as antimalarial drugs [7]. dosage regimen used in COVID-19 trials were chloroquine 500-1.000 mg per day for 7-10 days or hydroxychloroquine $400 \mathrm{mg}$ per day for 5-10 days [23].

\section{Adjunctive therapies}

Currently, due to the lack of specific drugs for COVID-19, the adjunctive therapies are the mainstay of treatment [19].

Monoclonal antibodies: Monoclonal antibodies can neutralize SARS-CoV they are directed against key inflammatory cytokines or other aspects of the innate immune response. Especially, IL-6 appears to be a key driver of this dysregulated inflammatory [19]. Though the risk of gastrointestinal (GI) perforation, hepatotoxicity, patients with thrombocytopenia and neutropenia, and infusion-related reactions which caution should be considered [21]. FDA suggests at tocilizumab, a monoclonal antibody IL-6 receptor antagonist, is used to treat cytokine release syndrome following chimeric antigen receptor T-cell therapy [19]. It works by binding the IL-6 receptor to prevent its activation and inhibits IL-6 signalling [22] . Based on these experiences; Tocilizumab is used in a small series of severe COVID-19 cases with early reports of success. A report of 21 patients with COVID-19 treated by using $400 \mathrm{mg}$ of tocilizumb, was associated with clinical improvement in $91 \%$ of patients measured by improved respiratory function, which most patients were only receiving one dose [19] and it plays as a stronger agent in the treatment of some serious COVID-19 cases with increasing level of IL-6 and spreading lung injuries [17]. Another IL6-receptor antagonist that is approved, other mAbs or immunomodulatory agents in a clinical trial in China or US including bevacizumab (anti-vascular endothelial growth factor medication), fingolimod (immunomodulator approved for multiple sclerosis), and eculizumab (antibody inhibiting terminal complement), [19]. Anakinra is an anti-IL-1 agent that has approved for managing of the autoimmune rheumatoid arthritis also showed 
satisfactoryresults [27]. Besides, scientists at the Dana-Ferber Cancer institute reported the human antibody isolation and used to block infection of the SARS-CoV. The 80R antibody is targeted to the spike glycoprotein and blocks the virus from binding to ACE2 receptors. Then, the antibody was tested in animal models which shows its protective function against acute lung injury. Such an antibody is used in passive immunization for the early treatment of the SARS-CoV infection. As that antibody was not fully protective, researchers from the National Cancer Institute later reported two new antibodies with improved affinity for the ACE2 receptor as compared to 80R. These mAbs, are m396 and S320. They neutralized viruses with exceptional potency that can be a good therapeutic or reagent to facilitate the development of a vaccine for SARS infections. Also, in a study by the US and Chinese researchers, mAbs were used in the treatment of MERS-CoV. In this study, the mAbs m336 neutralized the virus with exceptional potency and was reported to have great potential as a candidate therapeutic or as a reagent to facilitate the development of MERS-CoV vaccines in vitro [20]. As a result, the development of mAbs requires a certain period, which is difficult to achieve in a clinical application in a short time [3].

Immunoglobulin therapy: It is studied that convalescent plasma or hyperimmune immunoglobulins are used as adjunctive therapy for COVID-19. Antibodies from recovered patients may help with both free virus and infected cell immune clearance. As in the early phase, pathogens damage the targeted organs and lead to severe immunopathological damages. The passive immune antibodies can effectively and directly neutralize the pathogens, which reduce the damage of targeted organs and then block the subsequent immune-pathological damage. In 2009, a study on 93 patients of influenza A, revealed that those who receive convalescent plasma have less mortality rate than those who not receive the plasma. As a theory, the benefits of this therapy will appear after 7-10 days of infection, when viremia is at its peak, and the primary immune response has not yet occurred. Besides, the first reported uncontrolled case series of 5 critically ill patients with COVID-19 treated with convalescent plasma in China was recently published [19]. Thus, WHO emphasized that "convalescent plasma therapy is one of the most recommended potential therapies, and it has been used during other epidemic outbreaks." [18].

Corticosteroids: The most important point in the treatment of SARS-CoV-2 is to decrease the host inflammatory responses in the lungs, which may lead to acute lung injury and acute respiratory distress syndrome (ARDS). Although it might be decreased due to the existence of its adverse effects, including delayed viral clearance and increased risk of secondary infection, a study on MERS and SARS reported not any survival but also an association with delayed viral clearance from the respiratory tract and blood with high rates of complications including hyperglycemia, psychosis, and avascular necrosis. A study on patients with influenza pneumonia found that corticosteroids were associated with an increased risk of mortality. While a recent study in China on COVID-19 patients, treatment with methylprednisolone was associated with a decreased risk of death [22] therefore, because of its high pick ineffectiveness cautions against their routine use in patients with COVID-19 should be considered [19], as corticosteroids can be used to limit excessive lung damage due to inflammatory response. Methylprednisolone has already been used in COVID-19 patients in combination with antibiotics, oseltamivir, and oxygen therapy $40-80 \mathrm{mg} /$ day [2].

\section{Others}

Type 1 IFNs: Interferon (IFN) inhibits viral infection by directly interfering with viral replication by inducing both innate and adaptive immune response to infection [23]. Human immune system response is crucial to control viral replication which activation of innate interferon is necessary to avoid spreading viruses. Thus, their role in treatment is accepted in terms of their antiviral activity. Researchers in Frankfurt and Hong Kong recommended the antiviral activity of INF alfa, beta, and gamma, which among them IFN beta showed a good antiviral activity by inhibiting SARS-CoV replication. MERS-CoV is 50-100 times more susceptible than SARS-CoV to treatment with interferon alfa in vitro [20] . Also, previous studies have demonstrated that type 1 INFs can be used as an antiviral cytokine by inducing a large range of proteins to impair viral replication in targeted cells. As synergistic effects of leukocytic IFN alfa and IFN beta with Ribavirin against SARS-COV have been suggested in vitro [3]. Therefore, based on efficacy in SARS-CoV and MERS$\mathrm{CoV}$, a combination of antiviral and various subtype of interferon was studied for the efficacy in COVID-19 patients [23].

$\boldsymbol{R A S}$ inhibitors: According to the pathophysiology, ACE2 the content of the renin-angiotensin system (RAS) plays a significant role in the pathogenesis of SARS-COV2. Thus, possibly the blocking of the ACE2 via RAS inhibitors such as angiotensin-converting enzyme inhibitors (ACEIs) and angiotensin II receptor type 1 blockers (AT1RB) may have a major impact on choosing and designing of the new drugs [3].

Hypothiocyanite: Human has a natural protective network against infections. This system in central air tracts has a nit which involves the lactoperoxidase/ $\mathrm{H} 2 \mathrm{O} 2 / \mathrm{SCN}-(\mathrm{LPO} / \mathrm{H} 2 \mathrm{O} 2 / \mathrm{SCN}-)$ complex, and this complex induces the hypothiocyanite ion (OSCN-), [28]. The hypothiocyanite forms disulfide bonds via oxidation of thiol groups in proteins [29]. Thus, it can make alterations in surface proteins of respiratory tract infections such as viruses and prevent their binding to the target molecules. Disorders in synthesis and accumulation of viral nucleic acid and proteins is another possibly protective effect of the thiocyanate ion [30] Hence, and it strongly suggested the use of thiocyanate ion in controlling and managing of SARS-CoV-2 if proven according to the in-vitro and in vivo investigations [28].

Nitazoxanide: It is an antihelminthic agent, which has a broad antiviral activity and a good safety profile. It shows its antiviral activity against MERS and SARS-CoV-2, which these benefits require further study as a treatment option for SARS-CoV-2 [19].

Vitamin $C$ : is used in COVID-19 patients to reduce oxidative stress and inflammation in terms of its antioxidant activity [22]. Pneumonia is a complicated disorder of COVID-19 that can cause ARDS and serious and spread lung lesions. As Coronaviruses produce the free radicals and cytokine storm, they can worsen the situation to a life-threatening condition and multi organs dysfunction [31]. Therefore, antioxidant therapy, besides other managements, may help in controlling this 
situation. Vitamin $\mathrm{C}$ in a high dose is safe and effective [32] as well as it has a broad antiviral activity [33] which is another positive side of Vit $\mathrm{C}$ that shows it is an appropriate agent in the improvement of this condition. Thus, the application of Vit $\mathrm{C}$ as a monotherapy or combination therapy with other synergistic agents may play a crucial role in the managing of this situation [31].

\section{Conclusion}

This review summarized that SARS-CoV-2, which has similarity in terms of epidemiology and pathogeneses with SARS-CoV of 2003, has been spread from Wuhan, in China, which impacted the world most countries. It transmits from human to human mostly by respiratory droplets, directly or indirectly. It is suggested that for the first time, it transmitted from bats to humans, which confirms its zoonotic importance. The incubation period of novel coronavirus is within two weeks. The clinical signs include high fever, chills, cough, shortness of breath or difficulty in breathing, diarrhea, myalgia or fatigue, expectoration, and hemoptysis. People with a secondary disease or older people are at the front line of the risk. Though the world has not been faced with novel coronavirus infection before, thus there is not existed any specific medicine or vaccine to cure patients. But luckily, the similarity of the viral structure with that of SARS CoV makes it somewhat easy to tackle the pandemic novel coronavirus and commonly, the previously approved drugs are recommended for managing the COVID-19 patients. Recently, the FDA has approved the emergency use of an antiviral medicine named remdisivir in the treatment of COVID-19. However, scientists, medical doctors, and pharmaceutical organizations are working together to find a specific and safe drug for the treatment or a vaccine for the prevention and control of this worldwide viral disease.

\section{References}

[1] S. Navas-Martín and S. R. Weiss, "Coronavirus replication and pathogenesis: Implications for the recent outbreak of severe acute respiratory syndrome (SARS), and the challenge for vaccine development," Journal of Neurovirology, vol. 10, pp. 75-85, 2004. Available at: https://doi.org/10.1080/13550280490280292.

[2] M. Pal, G. Berhanu, C. Desalegn, and V. Kandi, "Severe acute respiratory syndrome coronavirus-2 (SARS-CoV-2): An update," Cureus, vol. 12, p. e7423, 2020.

[3] Y. Jin, H. Yang, W. Ji, W. Wu, S. Chen, W. Zhang, and G. Duan, "Virology, epidemiology, pathogenesis, and control of COVID-19," Viruses, vol. 12, p. 372, 2020. Available at: https://doi.org/10.3390/v12040372.

[4] M. Cascella, M. Rajnik, A. Aleem, S. Dulebohn, and R. Di Napoli, "Features, evaluation, and treatment of coronavirus (COVID-19)," Stat Pearls, 2021.

[5] World Health Organization, World health organization coronavirus disease (COVID-19) dashboard: World Health Organization, 2020.

[6] X. Li, M. Geng, Y. Peng, and L. Meng, "Molecular immune pathogenesis and diagnosis of COVID-19," Journal of Pharmaceutical Analysis, vol. 10, pp. 102-8, 2020. Available at: https://doi.org/10.1016/j.jpha.2020.03.001.

[7] M. I. I. Rabby, "Current drugs with potential for treatment of COVID-19: A literature review: Drugs for the treatment process of COVID-19," Journal of Pharmacy \& Pharmaceutical Sciences, vol. 23, pp. 58-64, 2020. Available at: https://doi.org/10.18433/jpps31002.

[8] L. Mousavizadeh and S. Ghasemi, "Genotype and phenotype of COVID-19: Their roles in pathogenesis," Journal of Microbiology, Immunology, and Infection, vol. 54, pp. 159-163, 2020. Available at: https://doi.org/10.1016/j.jmii.2020.03.022.

[9] H. Harapan, N. Itohd, A. Yufikae, W. Winardif, S. Keamg, H. Teh, j. Megawatii, D., Z. Hayatia, A. L. Wagnerl, and M. Mudatsir, "Coronavirus disease 2019 (COVID-19): A literature review," Journal of Infection and Public Health, vol. 13, pp. 667-673, 2020. Available at: https://doi.org/10.1016/j.jiph.2020.03.019.

[10] M. A. Faiq, A. Kumar, H. N. Singh, V. Pareek, R. Qadri, K. Raza, C. Kumari, R. K. Narayan, P. Kumar, and M. Kulandhasamy, "COVID-19: A review on molecular basis, pathogenic mechanisms, therapeutic aspects and future projections," 2020.

[11] F. Zhou, T. Yu, R. Du, G. Fan, Y. Liu, Z. Liu, J. Xiang, Y. Wang, B. Song, and X. Gu, "Clinical course and risk factors for mortality of adult inpatients with COVID-19 in Wuhan, China: A retrospective cohort study," The Lancet, vol. 395, pp. 10541062, 2020. Available at: https://doi.org/10.1016/s0140-6736(20)30566-3.

[12] D. Kaul, "An overview of coronaviruses including the SARS-2 coronavirus-molecular biology, epidemiology and clinical implications," Current Medicine Research and Practice, vol. 10, pp. 54-64, 2020. Available at: https://doi.org/10.1016/j.cmrp.2020.04.001.

[13] K. Martinez and Z. Villines, "What is the relationship between pneumonia and COVID-19? Medical News Today. Retrieved from https://www.medicalnewstoday.com/articles/pneumonia-and-covid-19," 2020.

[14] F. Di Gennaro, D. Pizzol, C. Marotta, M. Antunes, V. Racalbuto, N. Veronese, and L. Smith, "Coronavirus diseases (COVID19) current status and future perspectives: A narrative review," International Journal of Environmental Research and Public Health, vol. 17, p. 2690, 2020. Available at: https://doi.org/10.3390/ijerph17082690.

[15] W.-j. Guan, Z.-y. Ni, Y. Hu, W.-h. Liang, C.-q. Ou, J.-x. He, L. Liu, H. Shan, C.-1. Lei, and D. S. Hui, "Clinical characteristics of coronavirus disease 2019 in China," New England Journal of Medicine, vol. 382, pp. 1708-1720, 2020.

[16] K. Mcintosh, M. S. Hirsch, A. Bloom, and C. Disclosures, Coronavirus disease 2019 (COVID-19): Epidemiology, virology, clinical features, diagnosis, and prevention Hirsch, $M(E d)$. Waltham, MA: UpToDate, 2020.

[17] F. Peng, L. Tu, Y. Yang, P. Hu, R. Wang, Q. Hu, F. Cao, T. Jiang, J. Sun, and G. Xu, "Management and treatment of COVID19: The Chinese experience," Canadian Journal of Cardiology, vol. 36, pp. 915-930, 2020.

[18] T. Liang, "Handbook of COVID-19 prevention and treatment," The First Affiliated Hospital, Zhejiang University School of Medicine. Compiled According to Clinical Experience, vol. 68, 2020.

[19] J. M. Sanders, M. L. Monogue, T. Z. Jodlowski, and J. B. S. Cutrell, "Pharmacologic treatments for coronavirus disease 2019 (COVID-19): A review," Jama, vol. 223, pp. 1824-1836, 2020. Available at: https://doi.org/10.1001/jama.2020.6019.

[20] Clarivate, "Disease briefing: Coronaviruses," Canadian Journal of Anesthesia, pp. 1-81, 2020. 
[21] T. Smith, J. Bushek, A. Leclaire, and T. Prosser, COVID-19 drug therapy: Elsevier, 2020.

[22] S. G. V. Rosa and W. C. Santos, "Clinical trials on drug repositioning for COVID-19 treatment," Pan American Journal of Public Health, vol. 44, p. e40, 2020. Available at: https://doi.org/10.26633/rpsp.2020.40.

[23] N. Tidwong, P. Montakantikul, and W. Manosuthi, "Pharmacological treatment for the Novel Coronavirus disease 2019 (COVID-19 Infection)," Pharmaceutical Sciences Asia Review, vol. 47, pp. 97-103, 2020. Available at: https://doi.org/10.29090/psa.2020.02.020.0026.

[24] A. Rhoades, Remdesivir EUA letter of authorization vol. 564: FDA, Gilead Sciences, Inc, 2020.

[25] Y. Hewings-Martin and J. Collier, "FDA grant remdesivir emergency use for COVID-19 after turbulent week Medical News Today. Retrieved from https://www.medicalnewstoday.com/articles/fda-grant-remdesivir-emergency-use-for-covid-19-afterturbulent-week," 2020.

[26] The British Columbia COVID-19 Therapeutics Committee, "Unproven therapies for COVID-19, BC centre for Disease Control, BC Ministry of Health," pp. 1-30, 2020.

[27] Y. Shoenfeld, "Corona (COVID-19) time musings: Our involvement in COVID-19 pathogenesis, diagnosis, treatment and vaccine planning," Autoimmunity Reviews, vol. 19, p. 102538, 2020 . Available at: https://doi.org/10.1016/j.autrev.2020.102538.

[28] L. Cegolon, "Investigating hypothiocyanite against SARS-CoV-2," International Journal of Hygiene and Environmental Health, vol. 227, p. 113520, 2020. Available at: https://doi.org/10.1016/j.ijheh.2020.113520.

[29] Alexia, "ALX-009. Retrieved from https://www.alaxia-pharma.eu/alx-009/," n.d.

[30] L. Cegolon, C. Salata, E. Piccoli, V. Juarez, G. Palu, G. Mastrangelo, and A. Calistri, "In vitro antiviral activity of hypothiocyanite against A/H1N1/2009 pandemic influenza virus," International Journal of Hygiene and Environmental Health, vol. 217, pp. 17-22, 2014. Available at: https://doi.org/10.1016/j.ijheh.2013.03.001.

[31] A. Boretti and B. Banik, "Intravenous vitamin C for reduction of cytokines storm in acute respiratory distress syndrome. PharmaNutrition " vol. 12, ed: PUBMED, 2020, p. 100190.

[32] C. Hunt, N. K. Chakravorty, G. Annan, N. Habibzadeh, and C. J. Schorah, "The clinical effects of vitamin C supplementation in elderly hospitalised patients with acute respiratory infections," International Journal for Vitamin and Nutrition Research, vol. 64, pp. 212-219, 1994.

[33] C. W. Jungeblut, "A further contribution to vitamin C therapy in experimental poliomyelitis," The Journal of Experimental Medicine, vol. 70, pp. 315-332, 1939. Available at: https://doi.org/10.1084/jem.70.3.332s. 